\title{
Immunopotentiating activity of an herbal adjuvant: its benefits thereof
}

\begin{abstract}
Modern generation vaccines have poor immunogenicity and rely on adjuvants to potentiate their immunogenicity to generate a desired immune response. Adjuvants are macromolecular complexes that enhance immunogenicity of antigens in vaccines, thereby playing an important role in vaccine formulation. The present study aims to develop a de-novo herbal adjuvant derived from a medicinal plant growing in adverse climatic conditions at high altitude. The adjuvant was compared with CFA and Alum. Animals were immunized with native, recombinant and conjugated proteins. The humoral immune response was measured in terms of antibody titers. A relative contribution of Th1/Th2 type was evaluated in terms of IgG1 \& IgG2a isotyping and IFN- $\gamma$ \& IL4 levels. Splenocytes from mice immunized showed moderate T- cell recruitment in terms of cell proliferation. Antigen specific immunoglobulin levels were significantly enhanced by adjuvant at par with CFA \& Alum. Therefore, this adjuvant could be substitute for oil and alum adjuvants.
\end{abstract}

Volume 12 Issue 3 - 2019

\author{
Himanshi Tanwar, Sudipta Chanda, Monika \\ Jain Agrawal, Mallaeswara Rao E, Lilly Ganju \\ Defense Institute of Physiology and Allied Sciences, India
}

Correspondence: Lilly Ganju, Immunomodulation Laboratory, Defence Institute of Physiology and Allied Sciences, Lucknow Road,Timarpur, Delhi -I 10054, India, Tel 0 I I-23883 I6I, Fax 91-II-239|4790, Email Iganju@rediffmail.com

Received: May 10, 2019 | Published: June 19, 2019

Keywords: adjuvant, antibody, cytokine

\section{Introduction}

Contemporary vaccine development has focused on the design of subunit vaccines containing proteins or peptides from antigenic epitopes of infectious organisms. ${ }^{1}$ However, it is well known that the ability of vaccines to induce the desired immune response is dependent on adjuvant chosen for delivery, immunization schedule, route of immunization, delivery platform etc.

It is an established fact that adjuvants are able to generate robust immune response against recombinant antigens, synthetic peptides and weak immunogens. ${ }^{2-4}$ Adjuvants are also called immunopotentiators because of their ability to modulate immune response, which is directly proportional to the immune response they potentiate. The type and character of the induced immune responses are also influenced by the adjuvant., ${ }^{5,6}$ Therefore, the adjuvant has to be selected carefully for generating the desired immune response, for example, IgG class \&isotype profile, and the type of cytokines produced. ${ }^{7,8}$

There are many adjuvants available, but most of them are either toxic or do not generate immune response to the desired level. Commercially available adjuvant, Complete Freund's Adjuvant (CFA) is being used for last 50 years for producing antisera in animals. ${ }^{9,10}$ However, CFA has not been found suitable for human use as it causes local granuloma when administered. Calcium and Aluminium salts are known adjuvants registered for human use. Considerable number of adjuvants are currently under clinical trials, like immune stimulating complex, ${ }^{11}$ Quillaja Saponins, phospholipids, and hydrophobic or amphipathic protein. ${ }^{12}$ Overall, the activity of the adjuvant has been recorded with respect to magnitude and duration of antibody response. Other aspects of adjuvant activity are also being studied such as, influence on isotypes ${ }^{13,14}$ and local reaction at the site of injection.

In light of the above mentioned facts, we developed an adjuvant from Seabuckthorn, (SBT) (Hippophae Sp. L., Elaeagnaceae), a shrub, medicinal plantwidely spread at high altitude in cold desert trans Himalayan regions of India, China, Tibet \& Bhutan, has tremendous medicinal potential. ${ }^{15}$ SBT shrub is endowed with many nutritional and medicinal properties and is used for production of medically important fatty oils for commercial purpose. ${ }^{16}$ SBT leaves show marked antibacterial, antiviral, antitumor and wound healing properties. ${ }^{17,18}$ In addition SBT leaves have also been reported to have significant antioxidant, immunomodulatory, anti-hyperlipidemic, anti-myocardial and anti-fatty liver properties. ${ }^{19,20}$

\section{Results and discussion}

The adjuvanticity of DIP-HIP\# was compared with two commercially available adjuvants - CFA and Alum, in Balb/c mice. In case of TT immunized animals (S1A) titers with CFA and DIP-HIP administrations were significantly $(\mathrm{p}<0.01)$ higher (upto $1: 10,000,00)$ and more than alum administered animals $(1: 5,000,00)$. In case of DT immunization (S1B), CFA showed significantly $(\mathrm{p}<0.01)$ higher titer $(1: 10,00,000)$ as compared to DIP-HIP and alum. The titers with DIPHIP and alum were not significantly different $(1: 1,00,000)$. Antibody titers with all the three adjuvants were significantly higher $(\mathrm{p}<0.01)$ than PBS control animals.

Adjuvanticity of DIP-HIP was further compared with CFA and Alum using recombinant antigens DME, Hsp and Hsp-DME in Balb/c animals (S2). It was observed that in case of DME, both CFA and Alum showed significantly $(\mathrm{p}<0.01)$ higher titer $(1: 10,000)$ than DIPHIP (1:5000). Whereas, in case of Hsp, the titer with CFA and DIPHIP were significantly $(p<0.01)$ higher $(1: 10,00,000)$ than Alum (1: $5,00,000)$. At the same time with recombinant Hsp-DME conjugated protein, all the three adjuvants showed similar antibody titers $(1: 10,00,000)$ and significantly higher $(p<0.01)$ than PBS control animals. Balb/c Mice treated with antigens alone (without adjuvant), did not show detectable levels of antigen specific antibodies at any time point. The results indicated that antigen specific immunoglobulin levels were significantly enhanced by DIP-HIP and are at par with CFA \& Alum.

A relative measure of the contribution of Th1/Th2 type of immune response was also measured on the basis of isotypic variation. The $\operatorname{IgG1/IgG2a~isotype~ratio~was~measured~to~determine~the~relative~}$ measure of the contribution of Th1 and Th2 response. S4 depicts the isotypic antibody response in terms of optical density. The isotype response against antigens HSP, HSP-DME, TT and DT with all the 
three adjuvants showed significantly $(\mathrm{p}<0.01)$ higher response than PBS control. Alum showed significantly $(p<0.01)$ higher IgG1/ IgG2a ratio than CFA and DIP-HIP in case of Hsp, Hsp-DME and DT antigens, whereas in case of TT and DT immunization there was no significant difference between any of the three adjuvants. This demonstrates that DIP-HIP is able to stimulate both IgG1 and IgG2a antibodies in a pattern similar to that of CFA.

The adjuvanticity of DIP-HIP in Swiss albino was evaluated using TT and DT antigens (S3). Comparison between DIP-HIP and CFA showed a significantly $(\mathrm{p}<0.01)$ higher titer on DIP-HIP administration than CFA administered mice on immunization with TT (S3A) and DT (Fig. 3B). The efficacy of DIP-HIP was also evaluated in rabbits by using TT antigen. DIP-HIP showed significantly higher $(\mathrm{p}<0.01)$ titre $(1: 8,00,000)$ than CFA administered animals $(1: 5,00,000)(\mathrm{S} 3)$. The magnitude of the antibody response using different adjuvants varied with the strain and species of animals.

To further confirm the Th1 and Th2 type of immune response, IL-4 and IFN- $\gamma$ cytokines were also measured (S5A and S5B). The antigen-adjuvant administered groups produced significantly higher $(p<0.01)$ amount of IL-4 and IFN- $\gamma$ than control mice. All the mice administered with DIP-HIP and CFA showed significantly higher $(\mathrm{p}<0.01)$ IL-4 production than alum administered mice (S5A). Between DIP-HIP and CFA, in Hsp, TT and DT immunizations, CFA administered mice showed higher IL-4 production than DIP-HIP administered, whereas, in case of Hsp-DME, DIP-HIP showed higher IL-4 production than CFA. In case of IFN- $\gamma$, (S5B) mice administered with DIP-HIP and CFA showed significantly higher $(p<0.01)$ IFN- $\gamma$ production than Alum. Between DIP-HIP and CFA, in case of TT, there was no significant difference between the two, whereas in case of Hsp and Hsp-DME immunizations, DIP-HIP administration showed significantly higher $(\mathrm{p}>0.01)$ IFN $-\gamma$ production than CFA. Interestingly in case of DT, CFA showed significantly higher $(\mathrm{p}>0.01)$ IFN $-\gamma$ production than DIP-HIP. Animals immunized with Alum had highest IgG1/ IgG2a ratio as compared to CFA and DIP-HIP indicating that Alum induced primarily Th2 type of immunity which is in line with other studies [38-42]. On the other hand, CFA and DIP-HIP shifted the humoral immune response towards Th1 type by generating higher IgG2a antibodies. This data correlated well with expression of IFN- $\gamma$ and IL-4 cytokines.

To analyze T-cell proliferation, mice were immunized with different adjuvants and antigens, the splenocytes from individual mice were isolated (after the final booster) and activated in vitro with ConA and antigens, with which animals were originally immunized with. (S6) demonstrates that in case of DME, Hsp-DME and TT, Alum showed the highest proliferation, followed by CFA and DIP-HIP administered animals. Whereas in case of Hsp, CFA and Alum showed similar and significantly higher $(\mathrm{p}>0.01)$ proliferation response than DIP-HIP. Interestingly, in DT immunized animals DIP-HIP showed highest proliferation followed by Alum and CFA administrations. Animals immunized with CFA, Alum and DIP-HIP generated significant number of splenocytes that produced IL-4, indicating thereby that all adjuvants were capable of inducing T-cell proliferation. Importantly, DIP-HIP adjuvant supported the generation of Th1 response in splenocytes by inducing the production of IFN- $\gamma$. Our findings go well in line with the findings of Cooper et al. ${ }^{21}$ who have reported the activation of MHC-I molecule leading to CTL response against HIV using saponin as an adjuvant. Therefore, increase in IFN- $\gamma$ production by DIP-HIP particularly with Hsp-DME, which is a viral conjugated antigen, may indicate its capability of activating CTL response, whereas, most adjuvants are efficient in eliciting MHC-II response leading to antibody production.

The inflammatory response at the site of injection was recorded for each group of rabbits immunized through intramuscular route. In animals immunized with DIP-HIP, histological sections prepared from representative sites of injection demonstrated intact muscle architecture even after 24 and $48 \mathrm{~h}$ of immunization (S7). There was no evidence of hypersensitivity or granuloma. Gross and histological examination of other organs failed to reveal metastatic granulomas of the type reported for CFA. ${ }^{22}$ The generation of antibody titer varies with the route of immunization. DIP-HIP neither showed any significant change in the titer, nor resulted in any muscular damage of granuloma formation indicating, thereby that DIP-HIP injection is safe while injecting and completely retains the muscular architecture.

In brief, it can be concluded that, with DIP-HIP - both the magnitude and the type of immune response were affected. A good adjuvant should elicit cell mediated immunity as well as adequate level of humoral immunity, to confer protection against the infectious agents or toxins. An adjuvant should also generate good immune response in different strains and species using different immunization routes. DIPHIP enhanced both Th1 and Th2 type leading to both humoral and cell mediated immunity. DIP-HIP did not cause any significant change in the muscle architecture at the site of administration. Therefore, as compared to CFA which is known to cause inflammation, granuloma and necrosis, ${ }^{23}$ the administration of DIP-HIP was harmless to the muscle tissues. A complete analysis of the results of the present study indicate that DIP-HIP, a herbal adjuvant has the potential for developing into a potent and immuno-effective adjuvant and can be used as a substitute for both CFA and Alum.

\section{Conclusion}

Results indicated that antigen specific immunoglobulin levels were significantly enhanced by DIP-HIP \& were at par with CFA and Alum. Efficacy of DIP-HIP was also tested with recombinant proteins $\&$ synthetic peptides which are generally weak immunogens. DIP-HIP combined with recombinant proteins showed considerable antibody response. DIP-HIP enhanced both Th1 \& Th2 type. The magnitude of the antibody response using different adjuvants varied with strain \& species of animals. The different routes of immunization did not cause any difference in the antibody pattern, or any muscular damage. DIPHIP alone did not evoke any non-specific response on its own. DIPHIP herbal adjuvant is therefore having the potential to be used as a potent and immune-effective adjuvant. Experimental details relating to this paper are available online, alongside with Figures S1 to S7.

\section{Acknowledgments}

None

\section{Conflicts of interest}

Author declares there are no conflicts of interest.

\section{Reference}

1. Cunha-Neto E. MHC-restricted antigen presentation and recognition: Constraints on gene recombinant and peptide vaccines in humans. Braz J Med Biol Res. 1999;32(2):199-205.

2. Azmi F, Ahmad Fuaad AA, Skwarczynski M, et al. Recent progress in adjuvant discovery for peptide-based subunit vaccines. Hum Vaccin Immunother. 2014;10(3):778-796. 
3. Mohan T, Verma P, Rao DN. Novel adjuvant \& delivery vehicles for vaccines development: a road ahead. Indian J Med Res. 2013;138(5):779_ 795 .

4. Moyer TJ, Zmolek AC, Irvine DJ. Beyond antigens and adjuvants: formulating future vaccines. J Clin Invest. 2016;126(3):799-808.

5. Cox JC, Coulter AR. Advances in adjuvant technology and application. In: Yong WK, editor. Animal Parasite control utilizing Biotechnology. Boca Raton, FL: CRC Press Inc; 1992. p. 49-111.

6. Gupta RK, Rost BE, Relyveld E, et al. Adjuvant properties of aluminum and calcium compounds. In: Powell MF and Nowman MJ. Eds. Vaccine Design: The Subunit and Adjuvant Approach. New York: Plenum Publishing Corporation; 1955. p. 229-248.

7. Brewer JM, Alexander J. Studies on the adjuvant activity of non-ionic surfactant vesicles: adjuvant-driven Ig G2a production independent of MHC control. Vaccine. 1994;12(7):613-619.

8. Aramaki Y, Suda H, Tsuchiya S. IFN- $\gamma$ inductive effect of liposome as an immune-adjuvant. Vaccine. 1995;13(18):1809-1814.

9. FreundJ, Casals J, Hismer EP. Sensitization and antibody formation after injection of tubercle bacilli \& paraffin oil. Proc Soc Exp Biol Med. 1937;37(3):509.

10. Freund J. The effect of paraffin oil and mycobacteria on antibody formation and sensitization. Am J Clin Pathol. 1951;21(7):645-656.

11. Morein B, Sundquist B, Hoglund S, et al. Iscom, a novel structure for antigenic presentation of membrane proteins from enveloped viruses. Nature. 1984;308(5958):457-460.

12. Lovgren K, Morein B. The requirement of lipids for the formation of immune-stimulating complexes (Iscoms). Biotechnol Appl Biochem. 1988;10(2):161-172.

13. Ten Hagen TLM, Sulzer AJ, Kidd MR, et al. Role of adjuvants in the modulation of antibody isotype, specificity, and induction of protection by whole blood-stage Plasmodium yoelii vaccines. $J$ Immunol. 1993;151(12):7077-7085.
14. Brewer JM, Richmond J, Alexander J. The demonstration of an essential role for macrophages in the in vivo generation of IgG2a antibodies. Clin Exp Immunol. 1994;97(1):164-171.

15. SomenA, Tsering S, Shashi BS. Seabuckthorn (Hippophae sp. L.): New crop opportunity for biodiversity conservation in cold arid TransHimalayas. Soil and Water Conservation. 2010;9(3):1-204.

16. Bazaron EG, Tsybikova DT. Seabuckthorn is the remedy of Indo-Tibet medicine. Rastitel'nyye Resursy. 1978;14:67-69.

17. Shapiro DK, Garanovich IM, Anikhimovskaya LV, et al. Biochemical and morphological characteristics of prospective forms of seabuckthorn populations of North Azerbaijan. Rastitel'nyye Resursy. 1978;14:60-64.

18. Monika J, Sudipta C, Malleswara Rao E, et al. Effect of Hippophae Rhamnoides Leaf Extract against Dengue Virus Infection in U937 Cells. Virology \& Mycology. 2016;5:2.

19. Padwad Y, Ganju L, Jain M, et al. Effectofleaf extract of Seabuckthorn on lipopolysaccharide induced inflammatory response in murine macrophages. Int Immunopharmacol. 2006;6(1):46-52.

20. Geetha S, Sairam M, Singh V, et al. Anti-oxidant and immunomodulatory properties of seabuckthorn (Hippophaerhamnoides) -an in-vitro study. $J$ Ethnopharmacol. 2002;79(3):373-378.

21. Cooper PD. The selective induction of different immune responses by vaccine adjuvants. In: Ada GL, editor. Strategies in Vaccine Design. Austin: R.G. Landes Companypp; 1994. p. 125-158.

22. Lindblad EB. Adjuvants In: Stewart- Tull Des, editor. The theory and practical application of Adjuvants. Chichester: John Wiley and Sons Ltd; 1955. p.21-35.

23. Lai RP, Seaman MS, Tonks P, et al. Mixed adjuvant formulations reveal a new combination that elicit antibody response comparable to Freund's adjuvants. PloS One. 2012;11;7(4):e35083. 